Audiology

Neurotology
Audiol Neurotol 2019;24:38-48

DOI: $10.1159 / 000499154$
Received: June 20, 2018

Accepted after revision: February 25, 2019

Published online: April 17, 2019

\title{
Comparison of Place-versus-Pitch Mismatch between a Perimodiolar and Lateral Wall Cochlear Implant Electrode Array in Patients with Single-Sided Deafness and a Cochlear Implant
}

\author{
Jeroen P.M. Peters ${ }^{a, b} \quad$ Edwin Bennink ${ }^{c} \quad$ Gijsbert A. van Zanten ${ }^{a, b}$ \\ ${ }^{a}$ Department of Otorhinolaryngology and Head and Neck Surgery, University Medical Center Utrecht, \\ Utrecht, The Netherlands; ' UMC Utrecht Brain Center, Utrecht, The Netherlands; ' Image Sciences Institute, \\ University Medical Center Utrecht, Utrecht, The Netherlands
}

\section{Keywords}

Electric-acoustic pitch match · Place-versus-pitch · Cochlear implant · Frequency-place map - Single-sided deafness . Hearing loss · Perimodiolar electrode array · Modiolar hugging $\cdot$ Contour advance $\cdot$ Lateral wall electrode

\begin{abstract}
Background: In electric-acoustic pitch matching experiments in patients with single-sided deafness and a cochlear implant, the observed "mismatch" between perceived pitch and predicted pitch, based on the amended Greenwood frequency map, ranges from -1 to -2 octaves. It is unknown if and how this mismatch differs for perimodiolar versus lateral wall electrode arrays. Objectives: We aimed to investigate if the type of electrode array design is of influence on the electric-acoustic pitch match. Method: Fourteen patients ( $n=8$ with $\mathrm{Cl} 422$ + lateral wall electrode array, $n=6$ with $\mathrm{Cl} 1512$ + perimodiolar electrode array; Cochlear Ltd.) compared the pitch of acoustic stimuli to the pitch of electric stimuli at two test sessions (average interval 4.3 months). We plotted these "pitch matches" per electrode contact against insertion angle, calculated from high-resolution computed tomography scans. The difference between these pitch
\end{abstract}

\begin{tabular}{ll}
\hline KARGER & $\begin{array}{l}\text { (c) } 2019 \text { The Author(s) } \\
\text { Published by S. Karger AG, Basel }\end{array}$ \\
E-Mail karger@karger.com & This article is licensed under the Creative Commons Attribution- \\
www.karger.com/aud & $\begin{array}{l}\text { NonCommercial-NoDerivatives 4.0 International License (CC BY- } \\
\text { NC-ND) (http://www.karger.com/Services/OpenAccessLicense). } \\
\text { Usage and distribution for commercial purposes as well as any dis- } \\
\text { tribution of modified material requires written permission. }\end{array}$
\end{tabular}

matches and two references (the spiral ganglion map and the default frequency allocation by Cochlear Ltd.) was defined as "mismatch." Results: We found average mismatches of -2.2 octaves for the $\mathrm{Cl} 422$ group and -1.3 octaves for the $\mathrm{Cl} 512$ group. For any given electrode contact, the mismatch was smaller for the $\mathrm{Cl} 512$ electrode array than for the $\mathrm{Cl} 422$ electrode array. For all electrode contacts together, there was a significant difference between the mismatches of the two groups $(p<0.05)$. Results remained stable over time, with no significant difference between the two test sessions considering all electrode contacts. Neither group showed a significant correlation between the mismatch and phoneme recognition scores. Conclusion: The pitch mismatch was smaller for the perimodiolar electrode array than for the lateral wall electrode array.

(C) 2019 The Author(s)

Published by S. Karger AG, Basel

\section{Introduction}

A cochlear implant (CI) is an auditory prosthesis that partially restores auditory input in case of a deafened cochlea. A CI has an electrode array, which is surgically 
placed in the cochlea and distributes auditory information tonotopically: the lowest frequencies to the deepest inserted electrode contact (for all CI brands a center frequency of about $200 \mathrm{~Hz}$ ), the highest frequencies to the shallowest inserted electrode contact $(8,000 \mathrm{~Hz})$. The CI thereby mimics the tonotopy of auditory information of a normal ear. Patients with single-sided deafness (SSD) and a CI must fuse the electrical input from their CI with the acoustic input from their better ear. A possible mismatch in pitch between ears may have detrimental effects on the fusion of the signals from the two ears, which may limit performance [Buchman et al., 2014; Vermeire et al., 2015].

As the electrical stimulus activates spiral ganglion cells and not the organ of Corti, the classical place-pitch relation [Greenwood, 1990] has been amended for CI stimulation [Dorman et al., 2007; Stakhovskaya et al., 2007; Landsberger et al., 2015]. In pitch matching experiments in SSD patients implanted with a CI, the perceived pitch is 1 to 2 octaves lower than the pitch predicted by the amended place-pitch relation [Boëx et al., 2006; Dorman et al., 2007; Baumann and Nobbe, 2006; Schatzer et al., 2014]. Some of this mismatch may be due to the fact that in several studies [Boëx et al., 2006; Baumann and Nobbe, 2006; Dorman et al., 2007; McDermott et al., 2009; Schatzer et al., 2014; Vermeire et al., 2008; Vermeire et al., $2015]$ the determination of the electrode array's position was inaccurate, due to the use of planar X-ray imaging [Xu et al., 2000]. Furthermore, the better ear in these patients was moderately or severely impaired, resulting in possible distorted pitch perception [Gaeth and Norris, 1965].

In a previous paper, we described electric-acoustic pitch match experiments in ten patients with SSD and a CI [Peters et al., 2016]. In contrast to previous studies [Boëx et al., 2006; Dorman et al., 2007; Baumann and Nobbe, 2006; Schatzer et al., 2014], we determined the insertion angle of the electrode array using high-resolution computed tomography (HRCT) scans by a semiautomatic in-house-developed method [Bennink et al., 2017]. This method yields better knowledge of the exact intracochlear electrode array position and insertion angles for specific electrode contacts compared to planar $\mathrm{X}$-ray imaging. Additionally, we performed our experiments in a group of patients with near-normal hearing in their better ear, decreasing the risk of distorted pitch perception [Gaeth and Norris, 1965]. Even with these optimized methods, we also found a mismatch of about 2 octaves compared to the histological findings in human temporal bones by Stakhovskaya et al. [2007]. In our pre-

Place-versus-Pitch Mismatch in Patients with SSD and a CI vious paper, we also explored if the mismatch was of influence on the performance on a phoneme recognition test and found that they were not correlated [Peters et al., 2016].

In the current paper, we aimed to investigate if the type of electrode array design is of influence on the electricacoustic pitch match. We compared lateral wall electrode arrays and perimodiolar electrode arrays, with greater or smaller distance between the electrode array and spiral ganglion cells (located in the modiolus), respectively. The influence of the electrode array design in relation to performance has been investigated [Von Wallenberg and Briggs, 2014; Gibson and Boyd, 2016]: some authors concluded that a lateral wall electrode array led to better performance [O'Connell et al., 2016], whereas others found that proximity to the modiolus (i.e., perimodiolar) is correlated with better performance [Holden et al., 2013; Esquia Medina et al., 2013; Van der Beek et al., 2005]. Van der Beek et al. [2005] hypothesized that with the electrode array closer to the modiolus, "spatial selectivity" is enhanced: electrode contacts in close proximity to the spiral ganglion cells have a better chance of stimulating a more narrow tonotopic region of the cochlea than electrode contacts that are located farther away. Consequently, there is a better discrimination between electrode contacts, which leads to improved speech perception [Holden et al., 2013]. Additionally, we performed a re-test after the initial experiment to investigate if the results of the electric-acoustic pitch match experiments are stable over time, like they were in the experiments conducted by Vermeire et al. [2015].

\section{Methods}

A large part of the methods we used is identical to the methods that we detailed in Peters et al. [2016]. We will briefly repeat these methods and elaborate where new methods were used.

\section{Patients}

Fourteen patients with SSD and a CI participated in the current experiment, divided into two groups. The "CI422" group consisted of eight patients implanted with a CI with a lateral wall electrode array (Cochlear ${ }^{\circledR}$ CI422 Slim Straight electrode array). The "CI512" group consisted of six patients implanted with a CI with a perimodiolar electrode array (Cochlear ${ }^{\circledR}$ CI512 Contour Advance electrode array). All patients provided informed consent for participation in this study, which was ethically approved by the Institutional Review Board of the University Medical Center Utrecht (NL45288.041.13 and its later amendments). One of the inclusion criteria of the study is a pure tone average threshold in the better ear $\left(\mathrm{PTA}_{\mathrm{be}} ; 500,1,000,2,000\right.$, and 4,000 Hz) of maximum $30 \mathrm{~dB} H \mathrm{HL}$ in the better ear and of at least $70 \mathrm{~dB} \mathrm{HL}$ in the poor ear. 
Table 1. Patient characteristics

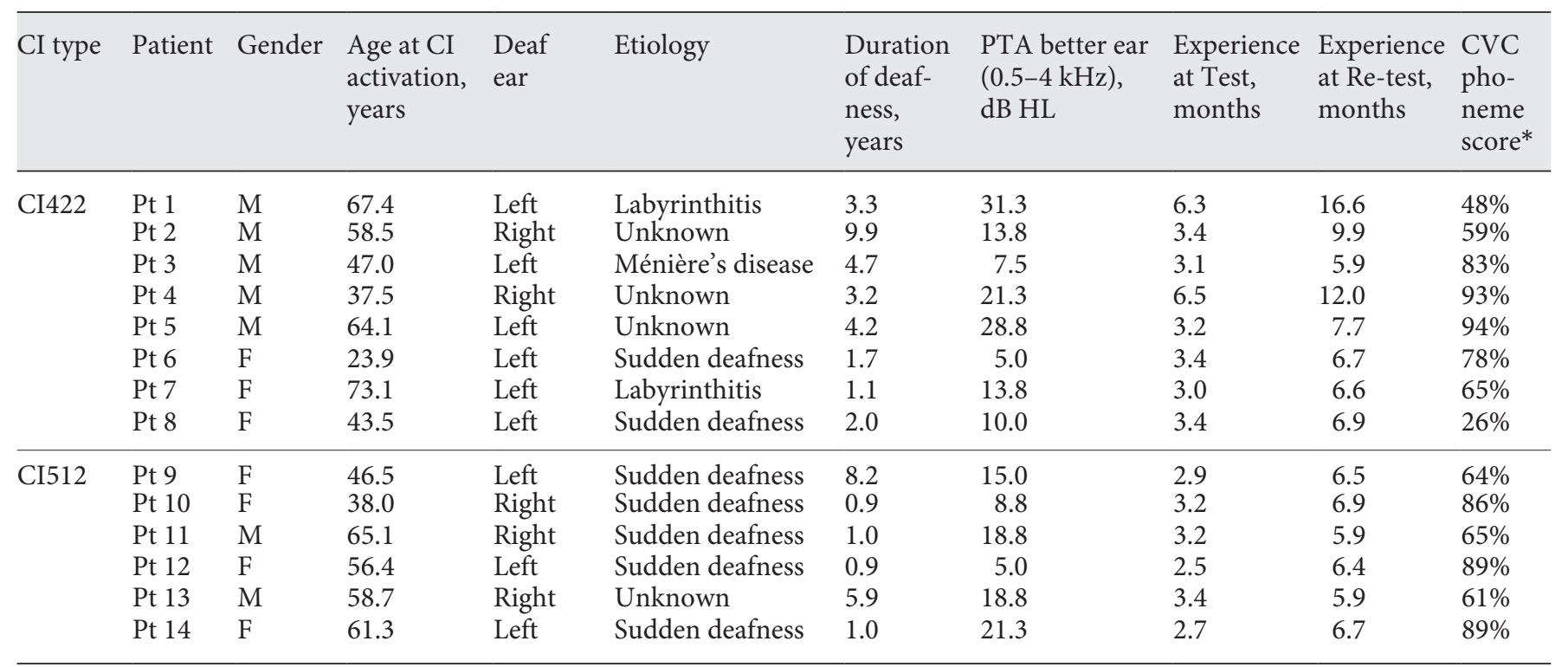

PTA, pure tone average $\left(500,1,000,2,000\right.$, and 4,000 Hz). ${ }^{*} \mathrm{CVC}$, consonant-vowel-consonant phoneme score at 3 months after CI activation for the CI ear only (better ear masked with masking noise). Patient 1 (Pt 1) had a PTA of the better ear of $30.0 \mathrm{~dB}$ HL at time of inclusion, but his hearing slightly deteriorated.

For details about other inclusion and exclusion criteria, see the study protocol [Peters et al., 2015]. All patient characteristics are summarized in Table 1 . All patients were tested twice, first after a median duration of 3.2 months after CI activation ("Test") and a second time after a median duration of 6.7 months after CI activation ("Re-test"). The average time between the Test and Re-test session was 4.3 months. Of the eight patients in the CI422 group, the data of the Test session of the first six patients were also presented in our previous paper [Peters et al., 2016]. In Figure 1, the average hearing thresholds for the better ear are displayed per group for all frequencies $(125-8,000 \mathrm{~Hz})$. There was no significant difference between the $\mathrm{PTA}_{\text {be }}$ of the CI422 group compared to the CI512 group (Mann-Whitney, $p=0.897$ ).

All patients were satisfied with their CI and wore the device often, except patient $(\mathrm{Pt}) 8$ (CI422 group with $\mathrm{Pt} 8$ : median use 8.8 h/day, without Pt 8: 12.1 h/day; CI512 group: $12.1 \mathrm{~h} /$ day). There was no significant difference between groups with or without Pt 8 (Mann-Whitney, $p>0.05$ ). Pt 8 reported that she could not get used to the "shrill" and "electric" sound of the CI, even after many attempts to personalize the frequency mapping, including switching off several electrode contacts. She performed poorer than expected (Table 1). Nevertheless, she did participate in Test and Retest experiments. One year after implantation, she became a nonuser.

\section{Cochlear Implants}

The Cochlear ${ }^{\circledR}$ CI422 has a Slim Straight electrode array, which is a lateral wall electrode array. The Cochlear ${ }^{\circledR}$ CI512 has a Contour Advance electrode array, which is a perimodiolar electrode array. Both electrode array types consist of 22 electrode contacts: electrode contact number 1 (E1) is located most basally (high fre- quency tones) in the cochlea, and E22 is located most apically (low frequency tones). The allocated center frequencies per electrode contact are identical for the CI422 and the CI512. The dimensions of both electrode array types and the frequency allocation per electrode contact are depicted in Table 2. Note that the CI512 Contour Advance electrode array $(11.70 \mathrm{~mm})$ is shorter than the CI422 Slim Straight electrode array $(18.75 \mathrm{~mm})$.

For some patients ( 2 patients in CI422 group, 2 patients in CI512 group), specific electrode contacts were switched off during CI rehabilitation because of high impedances. Consequently, the center frequencies of the remaining active electrode contacts differed from default because of the automatic reallocation (see online Suppl. Material 1 for frequency allocations per patient; see www.karger.com/doi/10.1159/000499154 for all online suppl. material). The geometric mean of the center frequencies per electrode contact of all patients is referred to as the "Cochlear reference line" in this paper.

\section{Stimuli and Experimental Design}

The experiments took place in an acoustically insulated, singlewalled and internally heavily damped room (IAC Acoustics $\mathrm{GmbH}$, Niederkrüchten, Germany), with noise reduction coefficient $>0.8$, inside a custom-built already quiet room. To be able to use the data from our previous experiment [Peters et al., 2016], we used the same electric and acoustic stimuli in this experiment. In short, we electrically stimulated five electrode contacts separately (E3, E7, E11, E15, E19) four times in all patients at a comfortable level (C-level of the most frequently used program). The electric stimulus consisted of a continuously repeated biphasic pulse train (pulse duration $25 \mu$ s, pulse repetition rate $900 \mathrm{~Hz}$, train duration $5,000 \mathrm{~ms}$ ) followed by a silent interval of $200 \mathrm{~ms}$. The acoustic
40 


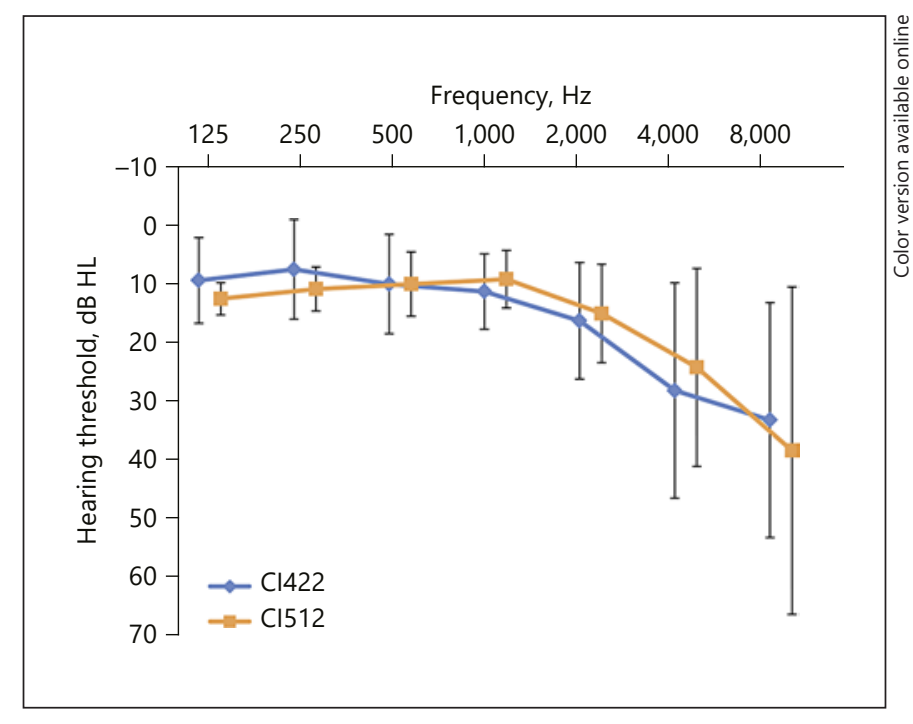

Fig. 1. Average pure tone hearing thresholds ( $\mathrm{dB} H \mathrm{HL})$ per group. There was no significant difference between the CI422 group and the CI512 group (Mann-Whitney, $p=0.897$ ). Error bars represent standard deviations.

stimuli consisted of pure tones, generated by a clinical audiometer and presented from a loudspeaker at $110 \mathrm{~cm}$ height (head level in seated position) and $130 \mathrm{~cm}$ distance in front of the patient. The manually presented acoustic stimuli had a minimum duration of $500 \mathrm{~ms}$. For the first run of measurements per electrode, we adjusted the level of a 1-kHz acoustic tone (median $60 \mathrm{~dB} \mathrm{HL}$, range 50-65 dB HL) to match it to the loudness of the electric stimulus in the $\mathrm{CI}$ ear (C-level of the most frequently used program). In this range (50-65 $\mathrm{dB} \mathrm{HL})$, the influence of loudness on pitch perception is negligible [Strange, 1955]. The task was to compare the pitch of two acoustic stimuli to the pitch of the electric stimulus and select the acoustic stimulus with greatest similarity in pitch. The difference between the two acoustic tones gradually decreased ( 2 octaves to $1 / 8$ octave), to end at the acoustic stimulus with a pitch "the most similar" to the pitch of the electric stimulus. We then calculated the geometric mean of the matched frequencies per electrode contact per patient ("pitch match").

\section{Insertion Angle of CI Electrode Array}

The intracochlear electrode array position was imaged using postoperative HRCT scans. Image acquisition and analysis are detailed in Bennink et al. [2017]. In short, each HRCT scan was filtered, and a $3 \mathrm{D}$ bounding box was manually positioned on the cochlea, in which the centerline of the electrode array was automatically tracked. The variation in CT values along this centerline was used to determine the electrode contact positions. The correlation of the CI geometry, as described in Table 2, with the variation in $\mathrm{CT}$ values was calculated at varying positions along the centerline. Since the CI512 is a pre-shaped perimodiolar implant, the correlation was also calculated for a varying stretch (0 to 15\%) with respect to its specified dimensions, whereas for the CI422, a compression ( 0 to $10 \%$ ) was allowed. The results were visually verified on a 4 -mm-thick reformatted projection of the scan, and another 
local optimum was chosen if the global optimum was found to be incorrect.

The transformation of the resulting CT coordinates to a cylindrical coordinate system (insertion angle, radius, elevation) requires fitting a plane through the basal turn of the cochlea and the manual selection of two reference points [Verbist et al., 2010a]. The selection of these two reference points, i.e., the top of the modiolus and the most lateral point of the horizontal semicircular canal, was carried out by an experienced radiologist. The top of the modiolus defines the center of the cylindrical coordinate system, whereas the line between the two reference points defines the $-34.6^{\circ}$ angle with respect to the position of the round window [Verbist et al., 2010b]

With knowledge of the insertion angle per electrode contact for all patients (see online Suppl. Material 1), we could calculate the predicted frequency using the Stakhovskaya histology results [Stakhovskaya et al., 2007; Table 3]. This predicted frequency is referred to as the "Stakhovskaya reference line" in this paper.

We expressed the intracochlear electrode array insertion as insertion angle instead of insertion depth, because the size of the cochlea may differ amongst patients [Stakhovskaya et al., 2007; Erixon et al., 2013; Van der Marel et al., 2014] and the intracochlear position of the electrode array may lead to different insertion angles for identical insertion depths.

\section{Data Analysis}

We compared the mismatch between the pitch matches of our patients to the Stakhovskaya reference line and the Cochlear reference line. We analyzed this per group (CI422 and CI512) and per experiment (Test and Re-test). A 2-sided one sample $t$ test was used to test if the pitch matches per electrode contact differed significantly from the reference lines. For between-group comparisons, the Mann-Whitney U test was used; for within-group comparisons, the Wilcoxon signed rank test was used.

Additionally, we checked if a correlation existed between the mismatch and the patient characteristics listed in Table 1 (age, duration of deafness, experience with $\mathrm{CI}, \mathrm{PTA}_{\text {be }}$ ). Finally, we also checked if there was a correlation between the mismatch and the performance on a speech recognition task (consonant-vowel-consonant (CVC) phoneme recognition scores 3 months after CI activation for the CI-ear only; better ear masked with speech-shaped noise, presented via an insert earphone, at a speech-to-masking level ratio of $-10 \mathrm{~dB}$ or worse) (see Table 1). The standard Dutch CVC phoneme recognition task was used, in which eleven CVC words with 33 phonemes per list are presented [Bosman and Smoorenburg, 1995]. The lists were presented in a free field condition at $65 \mathrm{~dB}$ SPL. Two lists were presented in order to improve measurement accuracy of the CVC score.

Data analysis was performed using SPSS Statistics software version 22 . A $p$ value of $<0.05$ was considered statistically significant.

\section{Results}

\section{Intracochlear Electrode Array Position}

Figure 2a shows the radius (distance from electrode contact to the modiolus axis) relative to the insertion angle per group. The CI512 electrode array is shorter than the CI422 electrode array (dimensions presented in Table 2 ) and thus shallower inserted in the cochlea. The median insertion angle of E22 for the CI512 group is $307.3^{\circ}$, whereas the median insertion angle of E22 for the CI422 group is $399.7^{\circ}$. The radius of the CI512 electrode array is smaller than the radius of the CI422 electrode array for corresponding electrode contacts, which can be attributed to the preformed curvature of the CI512 electrode array.

In Figure $2 b$, the elevation of the electrode contacts from the basal turn of the cochlea is plotted versus insertion angle per group. Again, we can observe that the CI512 electrode array is shorter and shallower inserted. Because of its shorter length, it does not reach as high into the second cochlear turn as does the CI422 electrode array (averaged maximum elevation of E22 for CI512 group: $0.37 \mathrm{~mm}$, for CI422 group: $1.06 \mathrm{~mm}$ ).

Insertion angles showed great variability in our population, not only between groups, but also between individual patients. The electrode array of Pt 8 (CI422 group) was inserted very shallow (insertion angle E22: $235.0^{\circ}$ ), resulting in $4-5$ electrode contacts positioned outside the cochlea. This may have contributed to her poor performance, dissatisfaction, and finally becoming a non-user. Because E3 was positioned outside the cochlea, we did not use the insertion angle of this electrode contact for this patient in our calculations. In contrast, the electrode array of Pt 14 (CI512 group) was positioned deep in the cochlea, with E22 positioned at $421.4^{\circ}$ (relatively deep for CI512 group). For all individual data on insertion angles per electrode contact, please see online Suppl. Material 1.

\section{Pitch Match}

In total, we presented 8,169 comparisons to fourteen patients in two experiments consisting of four runs on five electrode contacts, resulting in a mean ( \pm standard deviation) of $14.8 \pm 3.6$ comparisons per patient per run per electrode contact per experiment. For a closer inspection of all individual measurements, please see online Suppl. Material 1. Like in our previous experiment $[\mathrm{Pe}-$ ters et al., 2016], we noticed that the task was often difficult for patients. The difficulty of the task may have contributed to the observed large intra- and intersubject variability, which is comparable to our previous observations. As discussed in our previous report, we again observed no octave ambiguities (in a two-tone comparison, octave ambiguity is the identification by a listener of a higher harmonic as the fundamental tone, which means that a frequency at integer multiple value of fundamental fre- 
Fig. 2. a Average radius versus insertion angle per group. When inserted, the radius (distance from electrode contact to modiolus axis) of the CI512 electrode array is smaller than the radius of the CI422 electrode array at the same insertion angle. The electrode array of the CI512 is shorter than the CI422 electrode array (see also Table 2). Error bars represent standard deviations. b Average elevation versus insertion angle per group. The maximum elevation (from the basal turn of the cochlea) of the deepest inserted electrode contact (E22) is smaller for the CI512 electrode array than for the CI422 electrode array. The electrode array of the CI512 is shorter than the CI422 electrode array (see also Table 2). Error bars represent standard deviations.

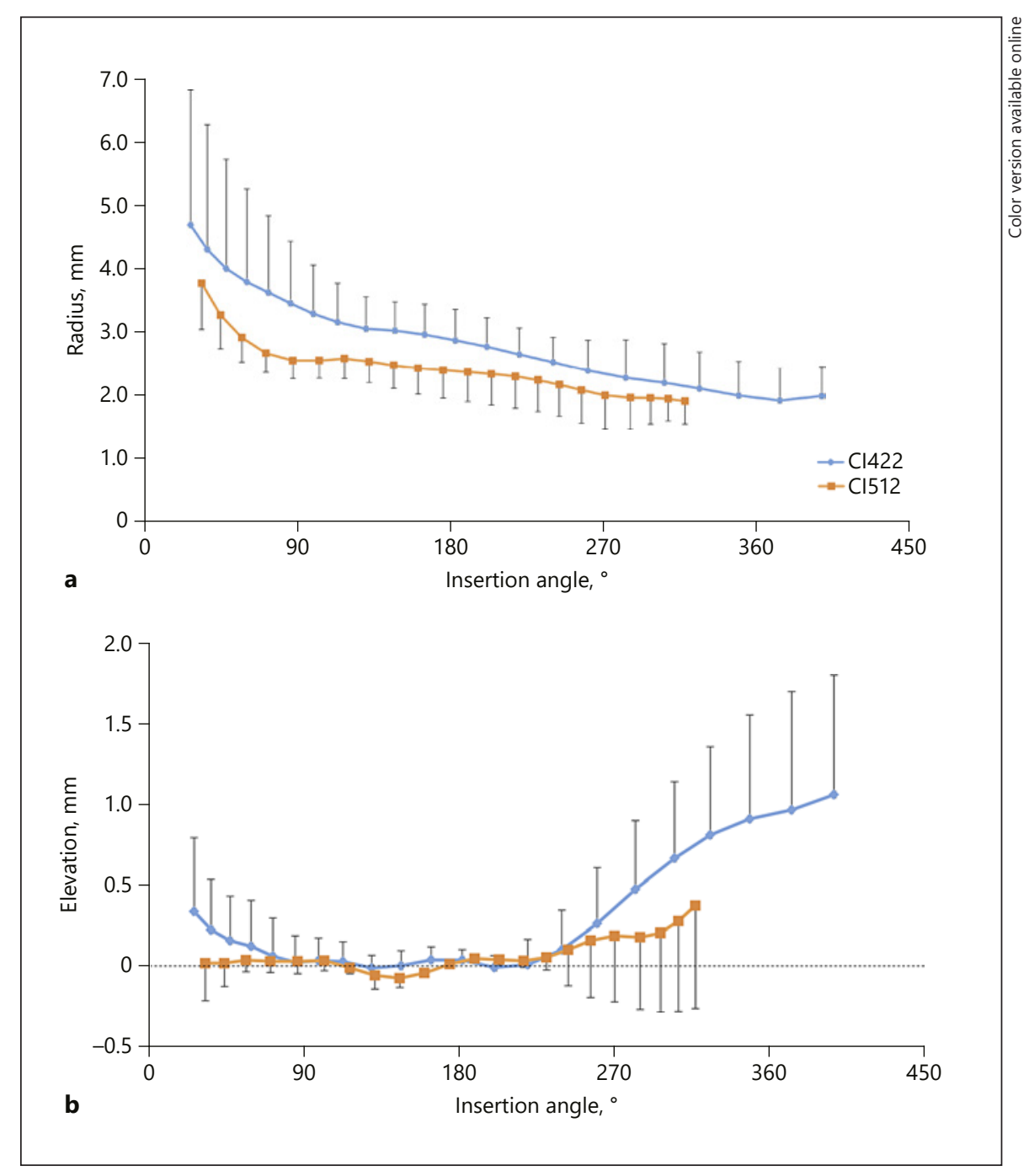

quency is confounded with the fundamental frequency itself), which is also concordant with other series [Baumann and Nobbe, 2006; Rader et al., 2016].

In Figure 3, the averaged pitch matches per group and per experiment are depicted for electrode contacts E3, E7, $\mathrm{E} 11, \mathrm{E} 15$, and E19. The logarithmic vertical axis shows the frequency and also denotes musical tones (e.g., A4 $=440$ $\mathrm{Hz}$ ), whereas the linear horizontal axis shows the insertion angle (degrees) of the electrode array. The two black lines represent the Stakhovskaya reference line and the Cochlear reference line. The averaged pitch matches for CI422 (blue) and CI512 (orange) are presented, split for experiments Test (solid line) and Re-test (dashed line). For the details of the coordinates of the two reference lines, see online Suppl. Material 1. As expected by the tonotopic organization of the cochlea and the program- ming of the CI, we observed that the frequency of the pitch matches decreased with increasing insertion angle (i.e., towards the apex of the cochlea). Again, we can observe that the insertion of the CI512 electrode array is shallower than the insertion of the CI422 electrode array. For both groups, there is a significant difference ("mismatch") between the averaged data points per electrode contact (Test and Re-test experiments) and the Stakhovskaya reference line (2-sided one sample $t$ test, $p<0.001$; for complete statistics, see online Suppl. Material 2). Compared to the Cochlear reference line, the CI422 group differs statistically significantly on all electrode contacts $(p<0.005)$, whereas the difference for the CI512 group is not statistically significant for E15 and E19 (at insertion angles of $230.17^{\circ}$ and $284.56^{\circ}$, respectively; $p>$ $0.05)$. Importantly, the average mismatch of the CI512 
Fig. 3. Average pitch match versus insertion angle per group. The averaged pitch matches (geometric mean, in $\mathrm{Hz}$ and musical notes, e.g., A $4=440 \mathrm{~Hz}$ ) per group (CI422 in blue, CI512 in orange) and per experiment (Test is solid line, Re-test is dashed line) and per electrode (E3, E7, E11, E15, E19) are plotted against the insertion angle (degrees). Also plotted are the Stakhovskaya reference line (striped black line) and the Cochlear reference line (dotted black line). For the $x$ coordinates of the Cochlear reference line, we used the averaged insertion angles per electrode contact for all patients. For readability of this figure, standard deviations are not included here. These can be observed in Figure 4.

Fig. 4. Mismatch expressed per electrode contact per group and experiment. The mismatch relative to the Stakhovskaya reference is plotted per electrode (data relative to Cochlear reference not shown). Data points are displayed separately for the CI422 and CI512 groups, as well as for the Test and Re-test experiments. For the CI422 group, there is no significant difference between Test and Re-test for any of the electrodes. For the CI512 group, E15 and E19 differ significantly between Test and Re-test, indicated with orange asterisks. For E11, there is a significant difference between the two groups for both Test and Re-test, indicated with black asterisks. For E15, the difference between the two groups is statistically significant for Retest, but not statistically significant for Test.
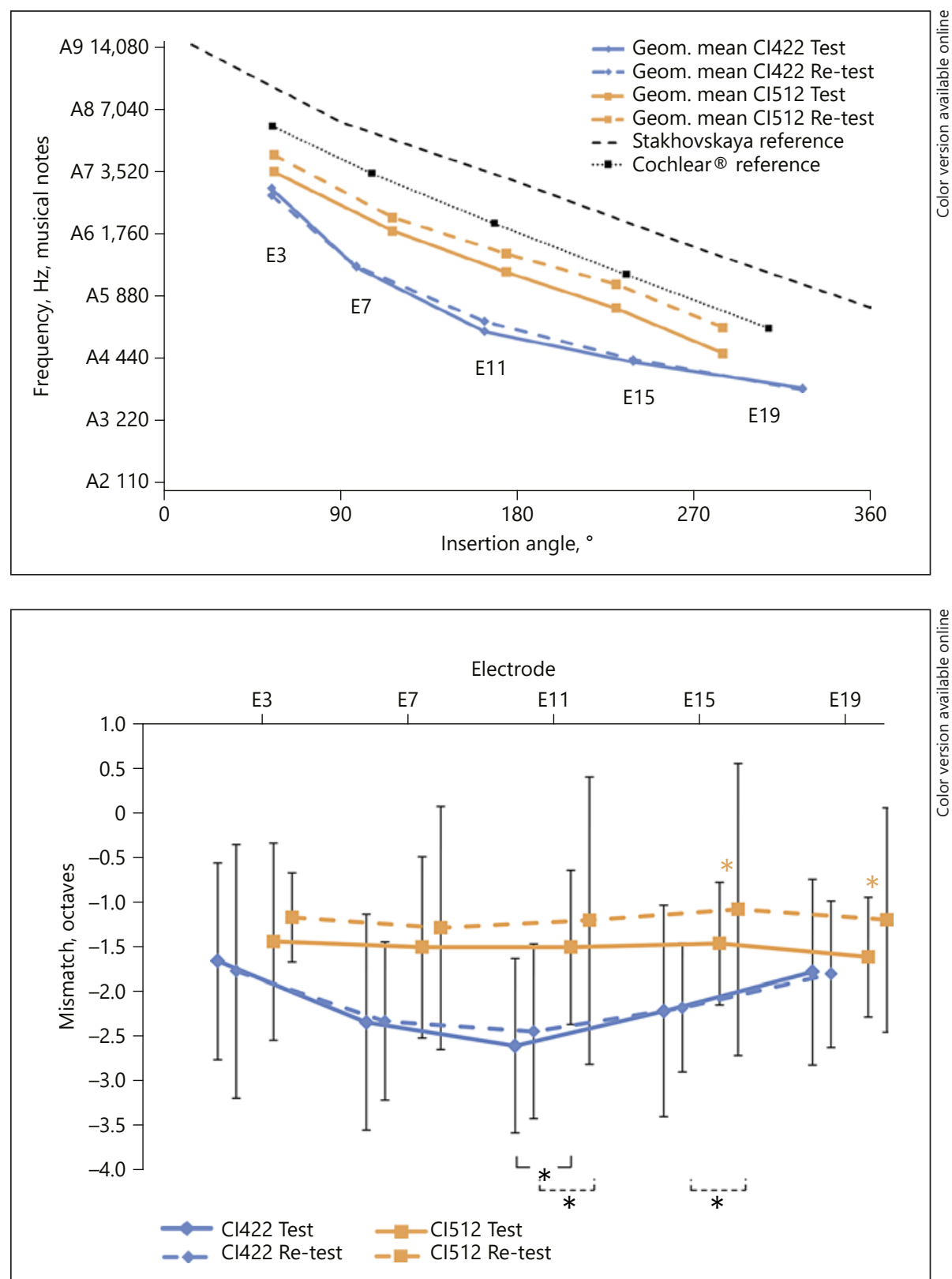

group is smaller than the average mismatch of the CI422 group.

\section{Mismatch}

To easily compare the two groups per electrode contact, we expressed the mismatch in octaves compared to the Stakhovskaya reference line (by definition 0 on the $y$ axis in Figure 4; data relative to Cochlear reference not shown). We calculated the average mismatch relative to the references for all electrode contacts (E3, E7, E11, E15, and E19) for both experiments (Test and Re-test) per group. The average mismatch relative to the Stakhovskaya reference is -2.2 octaves for the CI422 group and -1.3 octaves for the CI512 group (relative to the Cochlear reference: -1.4 octaves and -0.5 , respectively).

\section{Within-Group Comparisons}

For the CI422 group, there is neither a significant difference between Test and Re-test experiments for all electrode contacts together (Wilcoxon, $p=0.900$ ) nor for any 


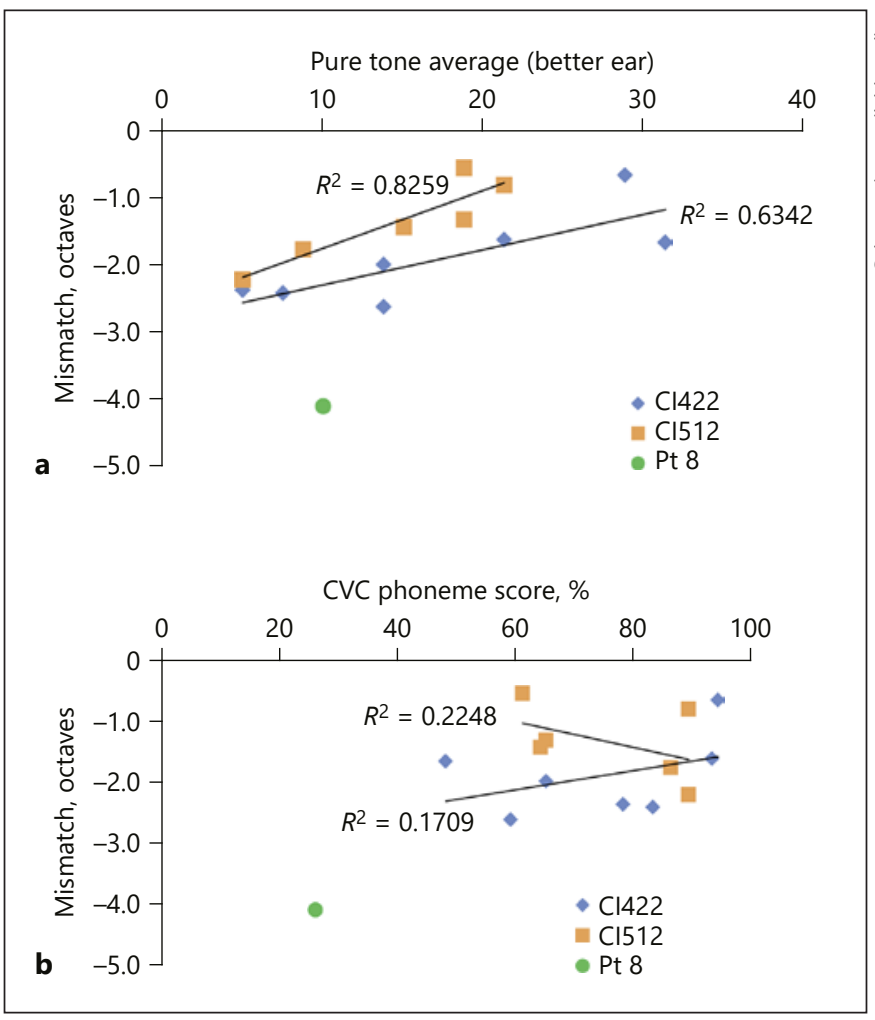

Fig. 5. a Mismatch (octaves) versus pure tone average of the better ear $\left(\mathrm{PTA}_{\mathrm{be}}\right)(\mathrm{dB} \mathrm{HL})$. There was a significant correlation between the observed mismatch and PTA $\mathrm{be}_{\mathrm{be}}$ for the CI512 group as well as for the CI422 group. Patient 8 ( $\mathrm{Pt} 8$, green dot) was excluded from this analysis. For all patients, the effect was also significant. b Mismatch (octaves) versus the score on a consonant-vowel-consonant (CVC) phoneme recognition task, 3 months after CI activation for the CI ear only. For both groups, there was no significant correlation between mismatch and the CVC phoneme score. Pt 8 (green dot) was excluded from this analysis. For all patients, the effect was also not significant.

of the individual electrode contacts $(p>0.208)$. For the CI512 group, there is a significant difference between the Test and Re-test experiments $(p=0.003)$. Looking at specific electrode contacts, E3, E7, and E11 do not differ significantly between Test and Re-test, but E15 and E19 do differ significantly between the two experiments $(p<$ 0.05), indicated with orange asterisks in Figure 4.

\section{Between-Group Comparisons}

For all electrode contacts together, there is a significant difference between the mismatches of the two groups (Mann-Whitney, $p<0.005$ ). When taking individual electrode contacts into account, there is no statistically significant difference between the groups for Test or Re-

Place-versus-Pitch Mismatch in Patients with SSD and a CI test $(p>0.14)$ for E3, E7, and E19. For E11, there is a significant difference between the two groups for both Test and Re-test $(p<0.05)$. For E15, the difference between the two groups is statistically significant for Re-test $(p=$ $0.013)$ but not for Test $(p=0.108)$, indicated with black asterisks in Figure 4.

\section{Correlations}

Due to the limited amount of data, correlations between patient characteristics and observed mismatch are of limited meaning, but our curiosity prevailed. We averaged the mismatch for all electrode contacts and for Test and Re-test experiments per patient. There were no correlations between the observed mismatches and patient characteristics age, duration of deafness, and experience with CI (maximum $R^{2}: 0.191, p>0.05$ ). There was a significant correlation between the observed mismatch and PTA $_{\text {be }}$ for the CI512 group $\left(R^{2}: 0.8259, p=0.012\right)$ as well as for the CI422 group $\left(R^{2}: 0.6342, p<0.05\right)$ (Fig. 5a). In this analysis we excluded outlier Pt 8 (green data point in Fig. 5a). For all patients (including Pt 8), the effect was also significant $\left(R^{2}: 0.3560, p=0.024\right)$. For both groups, there was no significant correlation between mismatch and the CVC phoneme recognition score (CI422 group $R^{2}: 0.1709, p>0.05$; CI512 group $R^{2}: 0.2248, p>0.05$ ) (Fig. 5b; Pt 8 [green data point] was excluded again). For all patients including Pt 8 , there was no significant correlation either $\left(R^{2}: 0.2737, p>0.05\right)$. For complete statistical analyses, see online Suppl. Material 2.

\section{Discussion}

In this study, we compared the results of electricacoustic pitch match experiments of patients with a CI 422 and a lateral wall electrode array and patients with a CI512 and a perimodiolar electrode array. Because of its shorter length, the CI512 electrode array is inserted shallower than the longer CI422 electrode array, but it is positioned closer to the modiolus axis due to its preformed curvature. We found average mismatches relative to the Stakhovskaya reference of -2.2 octaves for the CI422 group and -1.3 octaves for the CI512 group (relative to the Cochlear reference: -1.4 octaves and -0.5 octaves, respectively). For any given electrode contact, the mismatch to the two references was smaller for the CI512 electrode array than for the CI422 electrode array. For all electrode contacts together, there was a significant difference between the mismatches of the two groups. Results remained stable over time, with no significant difference 
between Test and Re-test experiments considering all electrode contacts. Neither group showed a significant correlation between the mismatch and CVC phoneme recognition score.

\section{Observed Mismatch}

Like in our previous report [Peters et al., 2016], the task appeared to be hard for the patients. They had difficulty matching the pitch of the acoustic stimulus to the pitch of the electric stimulus, partly because the stimuli sounded different. The difficulty of the task may have contributed to the observed large intra- and intersubject variability (see online Suppl. Material 1). Although pitch matching and pitch fusion experiments involve different tasks, it is conceivable that the outcome variability observed in this study is related to the earlier reported finding that patients can experience broad binaural pitch fusion between the CI ear and the better ear, equal to bilateral CI users, bilateral hearing aid users, and bimodal CI users [Reiss et al., 2014; Reiss et al., 2017a; Reiss et al., 2017b]. In spite of the task difficulty, the average results were stable over the observed time (average interval between Test and Re-test experiments was 4.3 months) for both groups. The good reproducibility is in line with the only other report investigating electric-acoustic pitch matches over time [Vermeire et al., 2015].

The average mismatch to the Stakhovskaya reference line was -2.2 octaves for the CI 422 group and -1.3 octaves for the CI512 group. The difference in mismatch may partly be attributed to the distance to the modiolus, which is, for any given electrode (Fig. 2a), shorter for a perimodiolar electrode array compared to a lateral wall electrode array. The range of observed mismatches is in line with our previous report ( -2.0 octaves for 10 SSD patients with CI422 [Peters et al., 2016]), and also concordant with other series evaluating various CI types in SSD patients [Boëx et al., 2006; Dorman et al., 2007; Baumann and Nobbe, 2006; Schatzer et al., 2014; Vermeire et al., 2015]. There are several other explanations proposed for the observed mismatch, including methodological shortcomings in the pitch matching procedures [Devocht et al., 2015], tonotopical reorganization after CI activation [McDermott et al., 2009; Vermeire et al., 2015], the electrical current between the electrode contacts and the extracochlear reference electrode following the trajectory of least resistance, which may differ from the histologically observed radial fiber trajectories [Peters et al., 2016; Stakhovskaya et al., 2007], and finally the degree of contralateral residual hearing [Vermeire et al., 2008]. One paper observed no mismatch between their electric-acoustic pitch match comparisons and the Stakhovskaya reference line [Carlyon et al., 2010]. They only used data that remained after so-called "sanity checks" of the experiments: presumably unreliable pitch match data were discarded. As we pointed out earlier in this paragraph and in our previous paper [Peters et al., 2016], we observed a large intra- and intersubject variability. We feel it is important to report all measurements and thereby present this variability and chose not to discard any data.

Remember that the audiograms of the two groups were similar (Fig. 1), and thus hearing threshold differences in the better ear cannot explain the difference in mismatch between the two groups. Psychoacoustic experiments in patients with asymmetric hearing loss showed that highfrequency hearing loss distorts pitch coding for pure tones with a frequency in the hearing loss range [Gaeth and Norris, 1965], resulting in pathologic hearing (diplacusis). In normal hearing subjects, the pitch of a pure tone may differ slightly between ears [Reiss et al., 2017a], but a fused single pitch is perceived in bilateral stimulation. Then, in the frequency range of the hearing loss, the pitch perceived in the poor ear is lower than the pitch perceived in the better ear and becomes lower as the hearing loss increases. Translated to our experiment, the pitch perceived in the better ear is lower when the hearing loss is bigger and concomitantly the mismatch will decrease, while the CI-evoked pitch for a specific electrode contact is constant. This could explain the correlation we detected between the mismatch and PTA ${ }_{\text {be }}$ (Fig. 5a), that is, with increasing hearing loss, the mismatch becomes smaller. We investigated a group of SSD patients with moderate (mainly high-frequency) hearing loss; when comparing our mismatch results to the results of groups with severe to profound (high-frequency) hearing loss [Boex et al., 2006; Dorman et al., 2007; Baumann and Nobbe, 2006; Schatzer et al., 2014; Vermeire et al., 2015], the mismatch found is about equal. Apparently, the effects of distorted pitch perception due to high-frequency hearing loss are small compared to those of the, still unknown, cause(s) of the mismatch.

\section{Intracochlear Electrode Array Position}

For both groups, we observed a large variance between maximum insertion angles of the most apical electrode contact (deepest and shallowest insertion of E22 in CI422 group: $537^{\circ}$ and $235^{\circ}$ [median $399.7^{\circ}$, whereas in CI512 group $421^{\circ}$ and $242^{\circ}$ [median $307.3^{\circ}$ ]). The large variation in insertion depth was also reported by Landsberger et al. [2015]. In contrast, they found a median insertion angle of the most apical electrode con- 
tact of the Contour Advance electrode array of $375^{\circ}$, which is deeper than the median insertion angle of $307.3^{\circ}$ in our study.

Some colleagues state that deeper insertion leads to improved speech perception [Zhou et al., 2016; Buchman et al., 2014], whereas others disagree [Van der Marel et al., 2015]. A similar debate continues about the position of the electrode array: some argue that a lateral wall electrode array leads to better performance [O'Connell et al., 2016], whereas others found that a perimodiolar position of the electrode array is correlated with better performance [Holden et al., 2013; Esquia Medina et al., 2013; Van der Beek et al., 2005]. In our study, we found a smaller pitch mismatch for the CI512 perimodiolar electrode array compared to the CI422 lateral wall electrode array.

\section{Methodological Considerations}

We presented the first series in which we investigated electric-acoustic pitch matches between two electrode array types in patients with SSD. Our patients have good hearing in their better ear, not resulting in a distorted pitch perception [Gaeth and Norris, 1965]. Furthermore, we determined the insertion angle based on HRCT scans by a semiautomatic method [Bennink et al., 2017], with which we could also determine radius and elevation of the electrode contacts.

There are also limitations of our approach. First, we did not match the loudness at all acoustic frequencies that we tested. This would have resulted in too much patient effort and thus compromise the willingness of patients to participate. Consequently, in our current results, the pitch matches may have been influenced by (mainly highfrequency) hearing loss in the better ear. However, we know that the influence of loudness on pitch perception is negligible in the tested range (50-65 dB HL) [Strange, 1955]. Second, there was a small sample size (CI422 group: $n=8$, CI512 group: $n=6$ ). Third, the intracochlear position of the electrode array in Pt 8 was very shallow, resulting in 4-5 disabled basal electrode contacts. Consequently, we did not consider E3 in this patient. Fourth, our semiautomatic method to determine intracochlear electrode array position did not allow us to determine if a scalar shift of the electrode array had occurred, which may also be of influence on performance and possibly mismatch [Shaul et al., 2018]. Finally, we tested performance using a CVC phoneme recognition score only. Since this is not a test for binaural hearing, other tests might be required to determine performance in this population.

Place-versus-Pitch Mismatch in Patients with SSD and a CI

\section{Implications of Findings}

In our previous report [Peters et al., 2016], we argued that the results of pitch matching experiments should be interpreted with caution: observed mismatches should not necessarily lead to adaptation of the default frequency allocation settings, since we did not observe a correlation between mismatch and performance. This was in contrast to evidence pointing in the direction that it is important to deliver auditory information at the correct cochlear place [Oxenham et al., 2004; Zhou et al., 2016; Reiss et al., 2016]. Possibly, reorganization of the neural system to the new frequency-place situation [Eggermont, 2017] may result in little impact of mismatch on performance. In our present study, we found that the pitch mismatch was smaller for the perimodiolar electrode array than for the lateral wall electrode array. However, our study is limited by a small number of patients per group. Therefore, we cannot draw conclusions regarding implications for clinicians and patients.

\section{Conclusion}

We found average mismatches relative to the Stakhovskaya reference of -2.2 octaves for the CI422 group and -1.3 octaves for the CI512 group (relative to the Cochlear reference: -1.4 octaves and -0.5 octaves, respectively). For any given electrode contact, the mismatch to the two references was smaller for the CI512 electrode array than for the CI422 electrode array. For all electrode contacts together, there was a significant difference between the mismatches of the two groups. Results remained stable over time (average interval between Test and Re-test experiments: 4.3 months), with no significant difference between Test and Re-test experiments considering all electrode contacts. Neither group showed a significant correlation between the mismatch and CVC phoneme recognition score.

References

Baumann U, Nobbe A. The cochlear implant electrode-pitch function. Hear Res. 2006 Mar; 213(1-2):34-42.

Bennink E, Peters JP, Wendrich AW, Vonken EJ, van Zanten GA, Viergever MA. Automatic Localization of Cochlear Implant Electrode Contacts in CT. Ear Hear. 2017 Nov/Dec; 38(6):e376-84.

Boëx C, Baud L, Cosendai G, Sigrist A, Kós MI, Pelizzone M. Acoustic to electric pitch comparisons in cochlear implant subjects with residual hearing. J Assoc Res Otolaryngol. 2006 Jun;7(2):110-24. 
Bosman AJ, Smoorenburg GF. Intelligibility of Dutch CVC syllables and sentences for listeners with normal hearing and with three types of hearing impairment. Audiology. 1995 SepOct;34(5):260-84.

Buchman CA, Dillon MT, King ER, Adunka MC, Adunka OF, Pillsbury HC. Influence of cochlear implant insertion depth on performance: a prospective randomized trial. Otol Neurotol. 2014 Dec;35(10):1773-9.

Carlyon RP, Macherey O, Frijns JH, Axon PR, Kalkman RK, Boyle P, et al. Pitch comparisons between electrical stimulation of a cochlear implant and acoustic stimuli presented to a normal-hearing contralateral ear. J Assoc Res Otolaryngol. 2010 Dec;11(4):625-40.

Devocht EM, Dees G, Arts RA, Smits JJ, George EL, van Hoof M, et al. Revisiting Place-Pitch Match in CI Recipients Using 3D Imaging Analysis. Ann Otol Rhinol Laryngol. 2016 May;125(5):378-84.

Dorman MF, Spahr T, Gifford R, Loiselle L, McKarns S, Holden T, et al. An electric frequencyto-place map for a cochlear implant patient with hearing in the nonimplanted ear. J Assoc Res Otolaryngol. 2007 Jun;8(2):234-40.

Eggermont JJ. Acquired hearing loss and brain plasticity. Hear Res. 2017 Jan;343:176-90.

Erixon E, Rask-Andersen H. How to predict cochlear length before cochlear implantation surgery. Acta Otolaryngol. 2013 Dec;133(12): 1258-65.

Esquia Medina GN, Borel S, Nguyen Y, AmbertDahan E, Ferrary E, Sterkers O, et al. Is electrode-modiolus distance a prognostic factor for hearing performances after cochlear implant surgery? Audiol Neurotol. 2013;18(6): 406-13.

Gaeth JH, Norris TW. Diplacusis in unilateral high-frequency hearing losses. J Speech Hear Res. 1965 Mar;8(1):63-75.

Gibson P, Boyd P. Optimal electrode design: straight versus perimodiolar. Eur Ann Otorhinolaryngol Head Neck Dis. 2016 Jun;133 Suppl 1:S63-5.

Greenwood DD. A cochlear frequency-position function for several species-29 years later. J Acoust Soc Am. 1990 Jun;87(6):2592-605.

Holden LK, Finley CC, Firszt JB, Holden TA, Brenner C, Potts LG, et al. Factors affecting open-set word recognition in adults with cochlear implants. Ear Hear. 2013 May-Jun; 34(3):342-60.

Landsberger DM, Svrakic M, Roland JT Jr, Svirsky M. The Relationship Between Insertion Angles, Default Frequency Allocations, and Spiral Ganglion Place Pitch in Cochlear Implants. Ear Hear. 2015 Sep-Oct;36(5):e20713.
McDermott H, Sucher C, Simpson A. Electroacoustic stimulation. Acoustic and electric pitch comparisons. Audiol Neurotol. 2009;14 Suppl 1:2-7.

O'Connell BP, Hunter JB, Gifford RH, Rivas A, Haynes DS, Noble JH, et al. Electrode Location and Audiologic Performance After Cochlear Implantation: A Comparative Study Between Nucleus CI422 and CI512 Electrode Arrays. Otol Neurotol. 2016 Sep;37(8):10325.

Oxenham AJ, Bernstein JG, Penagos H. Correct tonotopic representation is necessary for complex pitch perception. Proc Natl Acad Sci USA. $2004 \mathrm{Feb}$;101(5):1421-5.

Peters JP, Bennink E, Grolman W, van Zanten GA. Electro-acoustic pitch matching experiments in patients with single-sided deafness and a cochlear implant: is there a need for adjustment of the default frequency allocation tables? Hear Res. 2016 Dec;342:124-33.

Peters JP, van Zon A, Smit AL, van Zanten GA, de Wit GA, Stegeman I, et al. CINGLE-trial: cochlear implantation for siNGLE-sided deafness, a randomised controlled trial and economic evaluation. BMC Ear Nose Throat Disord. 2015 May; 15(1):3.

Rader T, Döge J, Adel Y, Weissgerber T, Baumann U. Place dependent stimulation rates improve pitch perception in cochlear implantees with single-sided deafness. Hear Res. 2016 Sep; 339:94-103.

Reiss LA, Eggleston JL, Walker EP, Oh Y. Two Ears Are Not Always Better than One: Mandatory Vowel Fusion Across Spectrally Mismatched Ears in Hearing-Impaired Listeners. J Assoc Res Otolaryngol. 2016 Aug;17(4): 341-56.

Reiss LA, Fowler JR, Hartling CL, Oh Y. Binaural Pitch Fusion in Bilateral Cochlear Implant Users. Ear Hear. 2018 Mar/Apr;39(2):390-7.

Reiss LA, Ito RA, Eggleston JL, Wozny DR. Abnormal binaural spectral integration in cochlear implant users. J Assoc Res Otolaryngol. 2014 Apr;15(2):235-48.

Reiss LA, Shayman CS, Walker EP, Bennett KO, Fowler JR, Hartling CL, et al. Binaural pitch fusion: comparison of normal-hearing and hearing-impaired listeners. J Acoust Soc Am. 2017 Mar;141(3):1909-20.

Schatzer R, Vermeire K, Visser D, Krenmayr A, Kals M, Voormolen M, et al. Electric-acoustic pitch comparisons in single-sided-deaf cochlear implant users: frequency-place functions and rate pitch. Hear Res. 2014 Mar;309: 26-35.

Shaul C, Dragovic AS, Stringer AK, O'Leary SJ, Briggs RJ. Scalar localisation of peri-modiolar electrodes and speech perception outcomes. J Laryngol Otol. 2018 Nov;132(11):1000-6.
Stakhovskaya O, Sridhar D, Bonham BH, Leake PA. Frequency map for the human cochlear spiral ganglion: implications for cochlear implants. J Assoc Res Otolaryngol. 2007 Jun; $8(2): 220-33$.

Strange $\mathrm{PH}$. The sense of pitch and local increase in threshold. J Physiol 1955 Aug 29;129(2): $225-36$.

van der Beek FB, Boermans PP, Verbist BM, Briaire JJ, Frijns JH. Clinical evaluation of the Clarion CII HiFocus 1 with and without positioner. Ear Hear. 2005 Dec;26(6):577-92.

van der Marel KS, Briaire JJ, Verbist BM, Muurling TJ, Frijns JH. The influence of cochlear implant electrode position on performance. Audiol Neurotol. 2015;20(3):202-11.

van der Marel KS, Briaire JJ, Wolterbeek R, SnelBongers J, Verbist BM, Frijns JH. Diversity in cochlear morphology and its influence on cochlear implant electrode position. Ear Hear. 2014 Jan-Feb;35(1):e9-20.

Verbist BM, Joemai RM, Briaire JJ, Teeuwisse WM, Veldkamp WJ, Frijns JH. Cochlear coordinates in regard to cochlear implantation: a clinically individually applicable 3 dimensional CT-based method. Otol Neurotol. 2010 Jul;31(5):738-44.

Verbist BM, Skinner MW, Cohen LT, Leake PA, James C, Boëx C, et al. Consensus panel on a cochlear coordinate system applicable in histologic, physiologic, and radiologic studies of the human cochlea. Otol Neurotol. 2010 Jul; 31(5):722-30.

Vermeire K, Landsberger DM, Van de Heyning $\mathrm{PH}$, Voormolen M, Kleine Punte A, Schatzer $\mathrm{R}$, et al. Frequency-place map for electrical stimulation in cochlear implants: change over time. Hear Res. 2015 Aug;326:8-14.

Vermeire K, Nobbe A, Schleich P, Nopp P, Voormolen MH, Van de Heyning PH. Neural tonotopy in cochlear implants: an evaluation in unilateral cochlear implant patients with unilateral deafness and tinnitus. Hear Res. 2008 Nov;245(1-2):98-106.

von Wallenberg E, Briggs R. Cochlear's unique electrode portfolio now and in the future. Cochlear Implants Int. 2014 May;15(sup1 Suppl 1):S59-61.

Xu J, Xu SA, Cohen LT, Clark GM. Cochlear view: postoperative radiography for cochlear implantation. Am J Otol. 2000 Jan;21(1):49_ 56.

Zhou X, Li H, Galvin JJ 3rd, Fu QJ, Yuan W. Effects of insertion depth on spatial speech perception in noise for simulations of cochlear implants and single-sided deafness. Int $\mathrm{J} \mathrm{Au}$ diol. 2017;56(sup2):S41-S48. 